\title{
Charged domain-wall dynamics in doped antiferromagnets and spin fluctuations in cuprate superconductors
}

\author{
J. Zaanen, M. L. Horbach, * and W. van Saarloos \\ Institute Lorentz for Theoretical Physics, Leiden University, P.O. Box 9506, NL-2300 RA Leiden, The Netherlands
}

(Received 18 January 1995; revised manuscript received 15 August 1995)

\begin{abstract}
Evidence is accumulating that the electron liquid in the cuprate superconductors is characterized by manyhole correlations of the charged magnetic domain-wall type. Here we focus on the strong-coupling limit where all holes are bound to domain walls. We assert that at high temperatures a classical domain-wall fluid is realized and show that the dynamics of such a fluid is characterized by spatial and temporal crossover scales set by temperature itself. The fundamental parameters of this fluid are such that the domain-wall motions dominate the low-frequency spin fluctuations and we derive predictions for the behavior of the dynamical magnetic susceptibility. We argue that a crossover occurs from a high-temperature classical to a lowtemperature quantum regime, in direct analogy with helium. We discuss some general characteristics of the domain-wall quantum liquid, realized at low temperatures.
\end{abstract}

\section{DOMAIN WALLS IN DOPED ANTIFERROMAGNETS}

Since the discovery of the cuprate superconductors the issue of hole doping in two-dimensional antiferromagnetic insulators has received considerable attention. Especially in the regime of low to moderate doping a number of unusual properties emerge. Here we want to suggest an interpretation of these phenomena in this regime in terms of holes bound to domain walls, separating antiferromagnetic regions - see Fig. 1. Fluctuations prevent these domain walls from freezing into an ordered state and we thus get a spatially and temporally disordered, liquidlike phase of the type sketched in Fig. 2. We associate the dynamics of this liquid state with the anomalous magnetic fluctuations of the cuprates.

We are motivated in part by recent studies of the $t-J$ model revealing tendencies towards the formation of such an inhomogeneous state over a large range of parameters. ${ }^{1}$ It was found, by exact diagonalization of small systems (18-20 sites) with four holes, that upon increasing $J / t$ a phase with domain-wall correlations appears before phase separation occurs. ${ }^{2}$ Earlier, these domain walls were found to be the classical (Hartree-Fock) saddle points of Hubbard models at
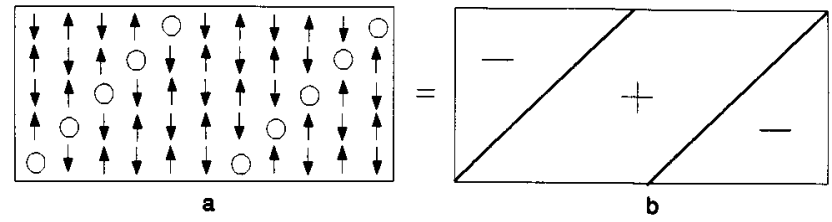

FIG. 1. Sketch of the domain-wall lattice as found in doped $\mathrm{La}_{2} \mathrm{NiO}_{4}$ (Refs. 8 and 9). (a) The arrows indicate the spins in the perovskite planes, centered on the $\mathrm{Ni}$ ions, and the circles indicate the holes. This corresponds with a reversal of the sign of the staggered order parameter over the domain walls where the holes are localized. In (b), the lines denote the position of the domain walls and the signs indicate the sign of the antiferromagnetic order parameter. Note that in $\mathrm{La}_{2} \mathrm{NiO}_{4}$ domain walls are oriented along the $(1,1)$ direction, while in the cuprates domain-wall correlations are supposedly along the $(1,0)$ and $(0,1)$ direction. finite doping, ${ }^{3,4}$ surviving in more sophisticated variational approaches such as the Gutzwiller ansatz ${ }^{5}$ and in fixed-node quantum Monte Carlo. ${ }^{6}$ We also mention that long-range Coulomb interactions are not as hazardous to this type of ordering as one would expect at first sight. It was recently shown that the mere electrostatics problem, combined with short-range attractive forces already favors the formation of striped charged phases. $^{7}$

Also some recent experimental developments give support to these domain-wall ideas. As we shall discuss later in more detail, these domain walls have recently been directly observed in doped $\mathrm{La}_{2} \mathrm{NiO}_{4},{ }^{8}$ which is a close relative of the cuprates. ${ }^{9}$ Indications for the existence of domain walls in lightly doped $\mathrm{La}_{2} \mathrm{CuO}_{4}$ were brought forward in the context of magnetic-susceptibility measurements. ${ }^{10}$ Finally, domain walls were proposed as a possible explanation for the Korringa behavior of the oxygen nuclear spin-lattice relaxation time in superconducting $\mathrm{La}_{2-x} \mathrm{Sr}_{x} \mathrm{CuO}_{4},{ }^{11}$ which is otherwise hard to understand in the light of the strongly incommensurate nature of the spin fluctuations in this material. $^{12}$

Motivated by the above considerations we study the dynamics of domain walls at finite temperatures, which should

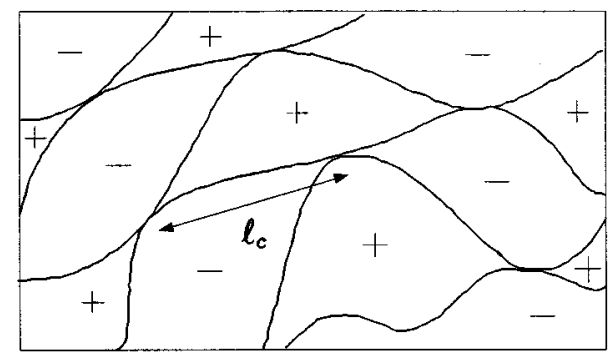

FIG. 2. Snap shot of the domain-wall liquid as is conjectured to exist in the cuprate superconductors also opposed to the ordered domain-wall state sketched in Fig. 1 . The characteristic length $\ell_{c}$ is indicated by the arrow and the sighns indicate the sign of the antiferromagnetic order parameter, as in Figs. 1 and 4. 


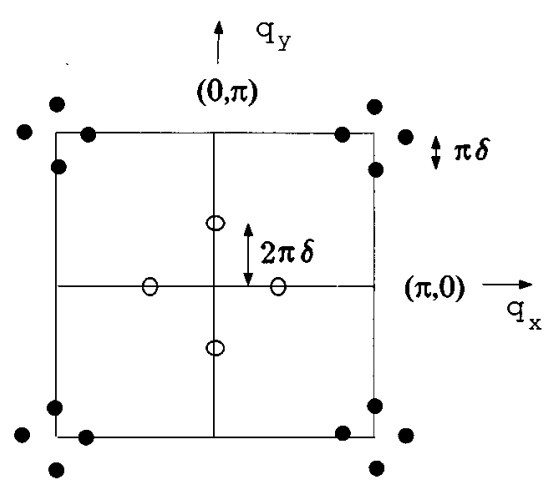

FIG. 3. Fourier transform of a domain-wall lattice as in Fig. 1, except that the lines are oriented along $(1,0),(0,1)$ directions in the square lattice as appears to happen in the cuprates. The spinlike first harmonics (filled dots) and the chargelike second harmonics (open dots) are indicated.

be in first instance reflected in the magnetic responses. We show that the dynamics of a system of fluctuating interacting quantum domain walls has a characteristic crossover frequency scale that is set by the temperature $\left(\hbar \Gamma \sim k_{B} T\right)$. Such a behavior is a prominent feature of the magnetic responses of the high- $T_{c}$ materials (e.g., neutron scattering, NMR), ${ }^{13}$ while it might also be held responsible ${ }^{14}$ for the anomalous properties of the metallic state in general. ${ }^{15,16}$

Domain walls yield a natural explanation for the momentum-space structure of spin fluctuations and its doping dependence as probed by neutron scattering. ${ }^{17}$ The Néel state at half-filling gives rise to magnetic Bragg peaks centered at $(\pi, \pi)$ in the Brillioun zone. In an ordered charge domain-wall array as shown in Fig. 1, the staggered order parameter has in addition a long-wavelength modulation with a period twice the domain-wall separation $d$ and the first harmonics are located at $[(1 \pm \delta) \pi, \pi]$ or $[\pi,(1 \pm \delta) \pi]$, as indicated in Fig. 3. Moreover it can be expected that the total length of the domain walls is proportional to the density of holes; it then follows that $\delta \sim 1 / d \sim x$, offering an explanation for the proportionality between hole density and incommensurability as found in the cuprates. ${ }^{17}$ If the domain-wall lattice is in a disordered state as sketched in Fig. 2, the Bragg peaks will broaden in momentum space with a width inversely proportional to the correlation length of the domain-wall lattice. Moreover, because the $(1,0)$ and $(0,1)$ directions are equivalent, the incommensurate peaks will show up in both directions in the zone. Although this is consistent with what is seen in neutron-scattering experiments, ${ }^{17,18}$ the experimental results do not unambiguously prove the existence of domain-walllike correlations. In order to experimentally demonstrate the existence of domain walls one would also have to observe the higher harmonics. ${ }^{4}$ For instance, if the spin modulation would be a single harmonic and in the transverse channel [Fig. 4(a)], like the spiral state of Shraiman and Siggia, ${ }^{19}$ one would already find the first harmonic while all other harmonics would have disappeared because the charge distribution would be uniform [Fig. 4(b)]. In fact, this situation is indistinguishable from the even simpler possibility that these "magnetic" fluctuations arise from the Fermi-liquid like excitations associated with the particular form of the Fermi

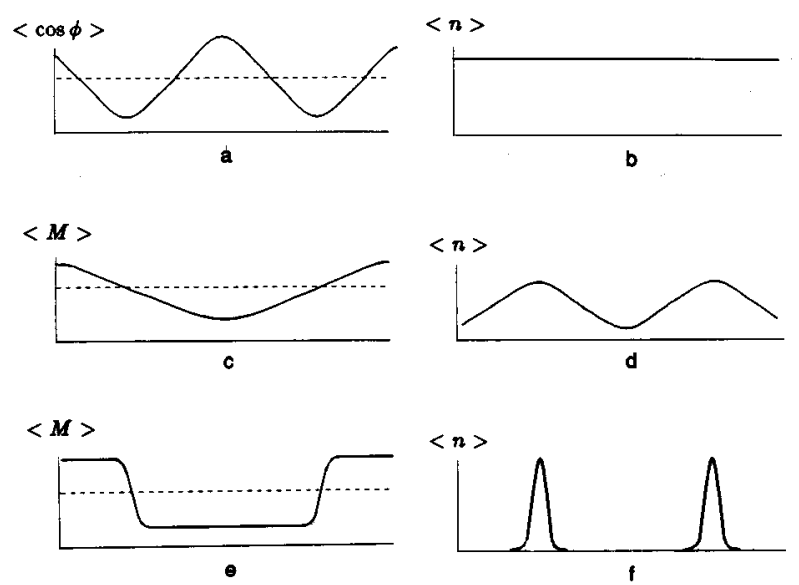

FIG. 4. The different possibilities for incommensurate orderings in doped magnetic insulators. The modulation of the spin direction (a) and the uniform charge distribution (b) of the spiral state. A single harmonic modulation of the size of the staggered order parameter (c) and the response of the hole density (d) if only two harmonics are present. The spin (e) and charge (f) components of the order parameter of the full domain-wall structure of Fig. 1.

surface. $^{20-22}$ On the other hand, a longitudinal (local moment) modulation of the spin density [Fig. 4(c)] would couple to the charge density and the latter modulation [Fig. $4(d)$ ] would give rise to a second harmonic with twice the magnetic incommensurability centered around the $\Gamma$ point. Finally, we have the "true" domain walls of Figs. 1, 4(e), and 4(f), characterized by third and higher harmonics representing the anharmonicities.

Although it is useful to picture a domain-wall as a string of holes as in Fig. 1(a), one should keep in mind that in reality the hole density is broadened ${ }^{3-6}$ over several lattice sites, as indicated schematically in Figs. 4(e) and 4(f). The wall positions drawn by lines in Fig. 2 therefore indicate the location of the maxima of the hole density or, equivalently, the points where the $z$ component of the spin order parameter $\left\langle M^{z}\right\rangle$ vanishes. When the wall width becomes of the order of the average wall separation, an array of walls will continuously transform into the modulated state of Figs. 4(c) and 4(d).

In fact, because it is near to impossible to measure the (valence) dynamical charge susceptibility directly, the only hope to see at least the second harmonics would be by the coupling of the electrons to the lattice. This is exactly what happens in both O- and Sr-doped $\mathrm{La}_{2} \mathrm{NiO}_{4}$ where the full harmonic structure associated with the structure of Figs. 1, 4(e), and 4(f) has been mapped out by neutron scattering. ${ }^{8}$ This material is rather similar to the cuprates. It is not only isostructural to $\mathrm{La}_{2-x} \mathrm{Sr}_{x} \mathrm{CuO}_{4}$, but a two-dimensional antiferromagnet at half filling with the same doping mechanism as well. However, the different physical properties (the nickelates are insulators at low temperatures) indicate that the holes in doped nickelates are more strongly localized. Evidence has been accumulating that this is primairily caused by a larger effect of the electron-phonon coupling. ${ }^{23,24}$

It has been argued that the electron systems in both cases are rather similar, except that both the larger lattice polaronic effects and the larger spin increase the mass of the walls, 
thereby suppressing the fluctuations, leading to the freeze out observed in the nickelates. ${ }^{9}$ In fact, the magnetic dynamics of the cuprates ${ }^{18,26}$ has some striking similarities with that of the $S r$-doped nickelate. Hayden et al. ${ }^{25}$ deduced from their neutron data on $\mathrm{La}_{1.8} \mathrm{Sr}_{0.2} \mathrm{NiO}_{4}$ that the $q$-integrated $\chi^{\prime \prime}(\omega)$ behaves as $\tanh (\omega / \Gamma)$, similar as in the cuprates. However, while the scale $\Gamma \sim T$ in the cuprates, $\Gamma$ drops to values much smaller than $T$ at $T \lesssim 100 \mathrm{~K}$ in $\mathrm{La}_{1.8} \mathrm{Sr}_{0.2} \mathrm{NiO}_{4}$. In a domainwall picture, the scale at which $\Gamma$ collapses might be identified with a domain-wall freezing temperature into a disordered phase (when $\Gamma$ is small the susceptibility has a typical glassy form). In contrast, in oxygen-doped $\mathrm{La}_{2} \mathrm{NiO}_{4}$ true long-range order is found at $T \lesssim 100 \mathrm{~K}$, corresponding with a lockin of the domain walls to the ordered excess oxygen striped phase. ${ }^{8}$

The scenario that we propose is that, while the domain walls form an ordered phase in the nickelates, they form a domain-wall liquid in the cuprates due to their stronger fluctuations. We will argue that there might be a hightemperature regime where thermal fluctuations dominate, characterized by the property that temperature sets the scale (Sec. III). From this analysis it follows that a crossover has to follow to a low-temperature regime dominated by quantum fluctuations, and we will identify the dimensionless parameter that governs this crossover, analogous to the de Boer parameter $^{27}$ for the heliums (Sec. IV). Finally, we will give a critical assessment of some (over)simplifications in our modeling (Sec. II) in the final Sec. V.

\section{DOMAIN WALLS AS VIBRATING STRINGS}

Let us identify some assumptions concerning the nature of the domain-wall liquid. In the next two sections we confine the discussion to a simplified model, which will be scrutinized in the final section:

(i) The domain walls behave as ideal, "Gaussian" strings. The internal degrees of freedom are completely parametrized by a single string transversal sound mode $\omega_{q}=c_{W} q$, where $q$ is the wave number along the string. The sound velocity is expressed in terms of a string tension ${ }^{28} \Sigma$ and mass density $\rho$ as

$$
c_{W}=\sqrt{\Sigma / \rho} .
$$

The string tension represents the effect of a change in energy associated with a change of length as a result of bending the wall. We notice that the commensuration gaps appearing in the spectrum of the collective modes ("phason") of the ordered phase ${ }^{29}$ are not present in the liquid.

(ii) We assume that dissipation is not important for the dynamics of a single string on the time scales of interest. Hence, isolated strings are characterized by coherent dynamics. This does not imply that isolated strings do not know about temperature. We assume that the only effect of temperature is to occupy the (coherent) sound modes of the strings which causes meandering. Dissipation sets in when strings start to collide. This is similar in spirit to kinetic gas theory, where dissipative hydrodynamics governs lowfrequency, long-wavelength behavior, while ballistic motion occurs on scales shorter than the collision length.

(iii) We assume that the low-frequency part of the spin excitation spectrum is dominated by the fluctuations of the domain-wall liquid. This is not a priori obvious, but can be justified as follows. If the undoped system is ordered, the spin system in the presence of walls can remain ordered if the effective spin-spin coupling across walls remains of the same order as the spin coupling inside the domains. In this case, the spin-wave velocity in the presence of walls will be largely unchanged from its undoped value. Alternatively, if the spin system is disordered but has a correlation length $\xi$ much larger than the domain-wall length $\ell_{c}$ defined below, spin waves still characterize the spin dynamics within the domains. In both cases, our scenario then assumes that the spin dynamics resulting from these modes follows adiabatically the domain-wall motion; the spin-wave velocity of spins inside the domains is assumed to be much larger than the wall velocity $c_{W}$. This assumption will be verified below. In this limit, the spin dynamics is induced by the motion of the walls, since the $z$ component of a spin flips when a wall passes, as can be verified from Figs. 1 and 4.

(iv) The wall-wall interactions are short ranged. We neglect both residual long-range Coulomb interactions, as well as the Casimir-type interactions mediated by the spin waves. $^{30}$

\section{CLASSICAL DYNAMICS OF A DOMAIN-WALL LIQUID}

The (classical) statistical mechanics of the model of Sec. II can be derived along similar lines as for two-dimensional incommensurate liquids formed by rare-gas atoms adsorbed on graphite, etc. ${ }^{31,32}$ We stress that this is not meant to imply that the present problem is physically the same. The microscopy is of course entirely different. Next to the fact that these charged domain walls are far more quantum mechanical (they are caused by local quantum fluctuations) than the structural domain walls, their density might obey a conservation law. Taking the on-string charge commensurability for granted as suggested by microscopic theories, ${ }^{3,4}$ their net density is proportional to the hole density and is therefore conserved. This is not the case in conventional incommensurate liquids and the present problem is actually somehat simpler.

Thermal fluctuations will cause the domain walls to meander. The central observation is that a new length scale emerges. ${ }^{33,34}$ Due to the wall wanderings, the walls will collide after a characteristic length $\ell_{c}$. At length scales smaller than $\ell_{c}$ one deals with isolated walls and at $\ell_{c}$ a crossover follows to a network of interacting walls. Assuming Gaussian walls and classical statistics, this length $\ell_{c}$ is easily determined. As is well known, the mean-square fluctuations of a single ideal string in two dimensions in the transverse $z$ direction between two points separated by a distance $\ell$ along the string, increases as

$$
\left\langle[z(\ell)-z(0)]^{2}\right\rangle \equiv\left\langle(\Delta z \ell)^{2}\right\rangle \simeq \frac{k_{B} T}{\Sigma} \ell .
$$

If we have an array of walls with average wall spacing $d$, due to the fluctuations the walls will on the average collide when $^{33}$

$$
\sqrt{\left\langle\left(\Delta z_{\ell_{c}}\right)^{2}\right\rangle} \simeq d
$$


The typical distance $\ell_{c}$ between collision points is then given by

$$
\ell_{c} \simeq \frac{\Sigma d^{2}}{k_{B} T}
$$

In other words, because the elementary objects are strings, we find naturally that a characteristic length scale (in fact a "microscopic" cutoff length) emerges which is inversely proportional to the temperature.

The length $\ell_{c}$ plays a central role in the classical equilibrium statistical mechanics of the fluctuating wall system. Our interest is in the dynamics of the wall liquid and in particular in the dynamics at the crossover scale. We suggest the following picture: at length scales smaller than $\ell_{c}$ the dynamics is that of stringlike vibrations of isolated walls with wavelengths shorter than $\ell_{c}$. At a length scale $\ell_{c}$ this crosses over to the dynamics of the many-wall problem. We directly obtain the characteristic time scale as follows. As long as the wavelength of the wall vibration is less than $\ell_{c}$ the dynamics is essentially the elastic stringlike dynamics of a single wall with $\omega=c_{W} q$. Hence, the energy scale $\hbar \Gamma$ for the crossover equals the value of $\hbar \omega$ at the wave number $q_{c},{ }^{35}$ i.e.,

$$
\hbar \Gamma=\hbar c_{W} \frac{q_{c}}{2}=\frac{\pi \hbar c_{W}}{\sum d^{2}} k_{B} T=\mu k_{B} T,
$$

where we have used Eq. (1) to introduce

$$
\mu \equiv \frac{\pi \hbar}{\rho c_{W} d^{2}} .
$$

In other words, "temperature sets the (frequency) scale," one of the hallmarks of the normal state of the cuprates, appears naturally in this domain-wall framework. Experimentally the dimensionless parameter $\mu$ is a quantity of order unity, ${ }^{13,18,26}$ such that the crossover scale is of order $k_{B} T$.

Let us present the argument more formally. We are seeking for the time scale of the relative motion of two collision points. Consider the Hamiltonian of an ideal classical string in two dimensions,

$$
H=\frac{1}{2} \int d \ell\left[\frac{p(\ell)^{2}}{\rho}+\Sigma\left(\frac{\partial z(\ell)}{\partial \ell}\right)^{2}\right]
$$

where $z(\ell)$ and $p(\ell)$ refer to the transverse position and momentum at position $\ell$ along the string, respectively. In terms of modes with wave number $q$ parallel to the string we then have

$$
H=\frac{1}{2} \int \frac{d q}{2 \pi}\left(\frac{\left|p_{q}\right|^{2}}{\rho}+\rho \omega_{q}^{2}\left|z_{q}\right|^{2}\right)
$$

with $\omega_{q}=c_{W}|q|$ and $q$ running from $-\pi / a$ to $\pi / a$. In the undamped case, each mode $z_{q}$ evolves with a frequency $\omega_{q}$; we then find for the unequal time correlation function, using the classical flucutation formula $\left\langle\left|z_{q}\right|^{2}\right\rangle=k_{B} T / \Sigma q^{2}$

$$
\begin{aligned}
\left\langle[z(\ell, t)-z(0,0)]^{2}\right\rangle= & \int_{-\pi / a}^{\pi / a} \frac{d q}{\pi}\left[1-\cos (q \ell) \cos \left(\omega_{q} t\right)\right] \\
& \times\left\langle\left|z_{q}\right|^{2}\right\rangle \\
= & \frac{2 k_{B} T}{\pi \Sigma} \int_{0}^{\pi / a} d q \frac{1}{q^{2}} \\
& \times\left(1-\frac{1}{2}\left[\cos q\left(\ell-c_{W} t\right)\right.\right. \\
& \left.\left.+\cos q\left(\ell+c_{W} t\right)\right]\right) .
\end{aligned}
$$

At equal times, $t=0$, one recovers the familiar result Eq. (2). At unequal times, however, this formula shows that the crossover time after which the relative motion of two points a distance $\ell$ apart becomes important, is $\ell / c_{W}$. This implies that the frequency scale above which this motion becomes important for two points a distance $\ell_{c}$ apart is just $\Gamma$. Likewise, the mean average fluctuations of two points a distance $\ell_{c}$ apart deviates significantly from the value $d$ after a time of order $\Gamma^{-1}$, so the collision frequency of nodes will also be of order $\Gamma$.

Note that the above discussion also clarifies our assumption (ii) of Sec. II concerning the timescale on which the wall dynamics is coherent, i.e., dissipationless: our assumption is that damping can be neglected on time scales shorter than $\Gamma^{-1}$, and the dissipation in the domain-wall liquid finds its origin in the collisions of the walls.

The significance of the scale $\Gamma$ is thus that it separates a high-frequency regime characterized by single-wall coherent dynamics and a low-frequency regime of dissipative, collective dynamics of the domain-wall liquid. Asserting that this has something to do with high- $T_{c}$ superconductors, $\Gamma$ has to be of order $k_{B} T$, i.e., $\mu$ in (6) has to be of order unity. It follows that the spin fluctuations as measured by inelastic neutron scattering should relate to the high-frequency regime down to the crossover frequency. On the other hand, the NMR relaxation rates measured at frequencies $\hbar \omega \ll k_{B} T$ should contain information concerning the hydrodynamic regime of the domain-wall liquid. Let us first deduce some simple ramifications for the spin-fluctuation spectrum in the frequency window of neutron scattering.

On time scales shorter than $\Gamma^{-1}$, we can think of the collision points as being fixed instantaneously. Nevertheless, it is still possible to excite this static "network" of domain walls because of the internal vibrations of the walls. At the collision points, the vibrational properties of the strings have to be perturbed because of the interactions with other strings. For simplicity we assume that these interactions have the effect of forcing nodes in the string vibrations at the collision points. The high-energy excitations can then be looked at as being the vibration modes of a collection of isolated string fragments with fixed end points. The mode density of a single string with length $\ell$ is given by

$$
g \ell(\omega)=\sum_{n=1}^{l / a} \delta\left(\omega-n \frac{\pi c_{W}}{\ell}\right) .
$$




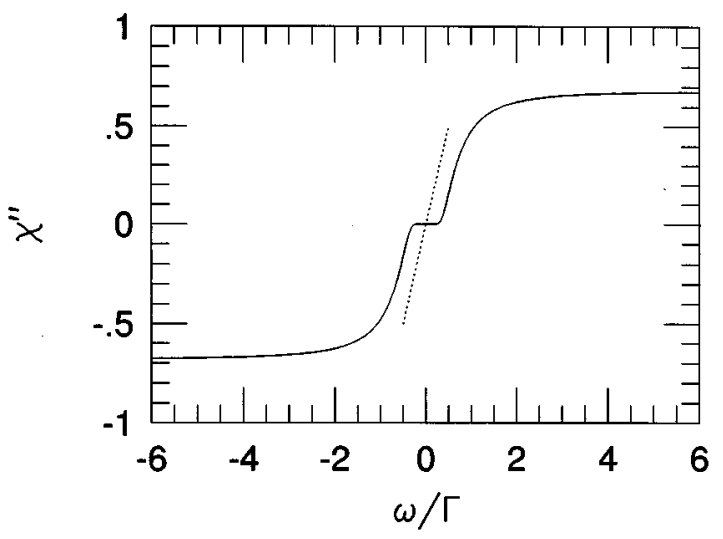

FIG. 5. The behavior of the $q$-integrated dynamical magnetic susceptibility, as deduced within the present approach. For $\omega \gtrsim \Gamma$, $\chi^{\prime \prime}$ is essentially flat and the response is dominated by the highfrequency dynamics of isolated walls. The solid line corresponds to the behavior as given by Eq. (13). The dashed line drawn for $\omega \leq \Gamma$ indicates that we expect the small frequency behavior, determined by the hydrodynamic response of the domain-wall network, to be essentially linear in frequency, as shown by Eq. (25).

The collision length $\ell_{c}$ is an average quantity. It is natural to assume a Gaussian distribution of collision lengths $\ell$ with a variance $\ell_{c}$,

$$
P(\ell)=\frac{1}{\ell_{c} \sqrt{2 \pi}} \exp \left[-\frac{1}{2}\left(\frac{\ell-\ell_{c}}{\ell_{c}}\right)^{2}\right] .
$$

The excitation spectrum averaged over this distribution is

$$
g(\omega)=\int d \ell P(\ell) g \ell(\omega)
$$

This translates into a (local) density susceptibility,

$$
\chi_{\rho}^{\prime \prime}(\omega)=\int d \ell P(\ell)\left[1+n_{b}(\omega)\right][g \ell(\omega)-g \ell(-\omega)],
$$

where $n_{b}(\omega)$ is the Bose factor and where we have left out an irrelevant form factor. For $\omega \gtrsim \Gamma$, where the single string dynamics underlying this formula is realistic, this expression give an $\omega$-independent susceptibility. This is illustrated in Fig. 5, where the dashed curves represent the contributions from the individual terms in the sum over $n$. Analytically, the flatness of $\chi_{\rho}^{\prime \prime}(\omega)$ for large frequencies can be most easily demonstrated by approximating the sum over $n$ by an integral over a continuous variable. Upon performing the $\ell$ integration first, one obtains

$$
\chi_{\rho}^{\prime \prime}(\omega)=(2 \Gamma)^{-1} \operatorname{sign}(\omega)\left[\operatorname{erf}\left(\frac{\Gamma / \omega+1}{\sqrt{2}}\right)-\operatorname{erf}\left(\frac{\Gamma / \omega-1}{\sqrt{2}}\right)\right]
$$

For frequencies $\omega \gtrsim \Gamma$, this expression rapidly approaches the constant value of $0.68 / \Gamma$, see Fig. 5. For small frequencies, Eq. (14) predicts the susceptibility to vanish exponentially fast for $\omega \ll \Gamma$; this is because the formula associates the low-frequency response with that of the long-wavelength vibrations of very long strings with $\ell \gg \ell_{c}$, whose probability of occurrence is exponentially small. However, the single string picture is not realistic for frequencies $\omega \lesssim \Gamma$ : the dynamical response is determined instead by the dynamics of the domain-wall network. However, these collective excitations cannot make up for the lack of single string excitations at low frequencies, so, as will be further clarified in our discussion of the hydrodynamical regime, $\chi^{\prime \prime}$ will decrease for small $\omega$. It is therefore expected that at $\Gamma, \chi_{\rho}^{\prime \prime}$ starts to decrease towards lower frequencies. In other words, we recover the typical "marginal" 16 behavior of the local dynamical susceptibility as measured by neutron scattering in the high- $T_{c}$ superconductors, ${ }^{18,26}$

$$
\chi^{\prime \prime}(\omega) \sim \tanh (\omega / \Gamma)
$$

with $\hbar \Gamma=O\left(k_{B} T\right)$.

The relationship between Eq. (7) and the spin-fluctuation spectrum is somewhat indirect, mediated by the modulation of the staggered order parameter by the domain-wall motions. However, under the assumption (iii) of the previous section, the spin dynamics is completely driven by the domain-wall motion. Therefore Eq. (7) is like the local dynamical spin suceptibility $\chi_{s}^{\prime \prime}(\omega)$ ( $q$-integrated dynamical magnetic susceptibility) modulo an overall normalization factor. The latter is easily determined. $\chi_{s}^{\prime \prime}$ is normalized by the total spin-flip probability per site for all spins in the system: $\int d \omega \chi_{s}^{\prime \prime}(\omega)=S^{2}=1 \mu_{B}^{2}$. However, only a fraction a/d of all spins can be flipped by a domain wall and therefore

$$
\int_{0}^{\infty} d \omega \chi_{s}^{\prime \prime}(\omega)=\left(\frac{a}{d}\right)^{2} \int_{0}^{\infty} d \omega \chi_{\rho}^{\prime \prime}(\omega) .
$$

Assuming that $\Gamma<c_{W} \pi / a$ (the high-frequency cutoff) we estimate for the spin susceptibility at high frequencies, in absolute units

$$
\chi_{s}^{\prime \prime}(\omega>\Gamma) \simeq \frac{a^{3}}{d^{2} c_{W} \pi} \mu_{B}^{2} / \mathrm{eV} .
$$

Also the spatial structure of the spin fluctuations can be addressed. The collision length $\ell_{c}$ is at the same time a dynamical disordering length for the domain-wall fluid. At low frequencies, the correlation length $\xi$ of the incommensurate magnetic order parameter (the inverse of the width of the incommensurate peaks in momentum space) should be

$$
\xi \simeq \ell_{c} \sim \frac{1}{T}
$$

We notice that this inverse scaling of the length scale with temperature is similar to what is found in the analysis of the critical regime of the quantum nonlinear $\sigma$ model where this scaling is associated with a dynamical critical exponent $z=1$. This is no surprise because the basic motive is similar: coherent quantum dynamics (instead of propagating string vibrations) emerging above a scale, which is proportional to temperature. $^{39}$

Being tied to the shortest length scale in the domain-wall fluid, the width of the incommensurate peaks should not change until one reaches the crossover regime where the coherent nature of the high-frequency dynamics starts to become important. This is illustrated in Fig. 6. The propagating 


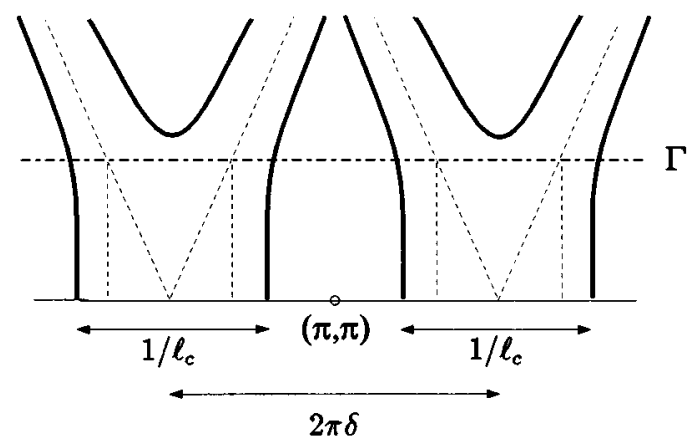

FIG. 6. The appearance of the dynamical magnetic susceptibility $\chi^{\prime \prime}$ both as function of wave vector $\vec{q}$ [on a line connecting the first harmonics around $(\pi, \pi)]$ and frequency $\omega$. The dashed lines indicate the string mode "cones" originating from the ordering wave vectors in the striped phase, if interaction effects would be neglected. Remnants of this dispersive behavior are found above the characteristic frequency scale $\Gamma$, while below this scale only the disordering length $\ell_{c}$ matters.

string excitations would form spin-wave-like "cones" originating at the incommensurate points if the domain walls were ordered, modulo possible collective effects at low frequencies because of wall-wall interaction energies. One should recover this $\vec{q}$ dependence at frequencies larger than $\Gamma$ in the liquid as well except that these excitations are broadened by $\Delta q_{c} \sim \pi / \ell_{c}$, because of the finite mean free path for the vibrations on the domain-wall "network." The result of this construction (Fig. 6) shows a striking similarity with the form of the dynamical magnetic susceptibility as function of $\vec{q}$ and $\omega$ as measured by Aeppli and co-workers ${ }^{38,18}$ using neutron scattering. In our picture, the momentum independence of the dynamics for frequencies less than $\Gamma$ reflects the mean-free path $\ell_{c}$ in the disordered domain-wall network, while at frequencies larger than $\Gamma$ the fluctuations gain a dispersive component coming from the single-wall dynamics.

From these simple considerations, quantitative predictions follow for the behavior at the crossover scale. (i) The frequency where the legs come together should increase with decreasing temperature (opposite to $\Gamma$ !) and the "crotch" should occur at a similar frequency as to where the crossover occurs in the $q$-integrated response (Fig. 6). (ii) The width of the "legs" ("magnetic correlation length") should increase roughly proportional to $\Gamma$ (or temperature), signaling the existence of the string sound velocity.

Although more data are needed to prove or disprove these predictions, the existing neutron data do not contradict this picture and the latter can be used to show that the numbers make sense. In our approach the magnetic correlation length $\xi$ is of order of the collision length $\ell_{c}$. Using Eq. (4) and $\xi \simeq 25 \AA$ at $35 \mathrm{~K}$ in $\mathrm{La}_{2-x} \mathrm{Sr}_{x} \mathrm{CuO}_{4},{ }^{18}$ while $d \simeq 10 \AA$ we find the string tension $\Sigma=1 \mathrm{meV} / \AA$. Then, for $\mu$ according to Eq. (6) to be of order unity, we obtain for the domain-wall mass density and sound velocity

$$
\begin{gathered}
\rho \simeq 10 m_{e} / a, \\
\hbar c_{W} \simeq 0.1 \mathrm{eV} \AA .
\end{gathered}
$$

Here we have written $\rho$ in terms of the electron mass $m_{e}$ and the lattice parameter $a \simeq 4 \AA$ of the cuprate layers. The sound velocity $c_{W}$ sets the energy scale of the wall vibrational modes and we find indeed that the domain-wall liquid is an order of magnitude softer than the spin system at halffilling, since the spin-wave velocity is of the order of $1 \mathrm{eV} \AA$ $/ \hbar$ [Sec. II, assumption (ii)]. More precisely, we can use Eq. (16) to estimate $\chi_{s}^{\prime \prime}(\omega>\Gamma) \approx \mathscr{Q}\left(1 \mu_{B}^{2}\right) / e V$, in agreement with experiment, and sufficient to "mask" the spin waves at low frequencies. Finally, notice that the mass density is of order of a few electronic masses.

Let us now turn to the low-frequency regime of the dynamics of the domain-wall liquid. Of primary interest is the low-frequency end of the dynamical magnetic susceptibility as probed by the $\mathrm{Cu}$ NMR relaxation rate, ${ }^{40}$

$$
\frac{1}{T_{1}} \sim k_{B} T \int d q^{2} S(q) \frac{\chi^{\prime \prime}\left(q, \omega_{0}\right)}{\omega_{0}}
$$

with $\omega_{0} \sim 10^{7} \mathrm{~Hz}$ (nuclear Larmor frequency) and $S(\vec{q})$ a form factor, a smooth function of $\vec{q}$, which is at maximum at the incommensurate wave vectors if the domain picture applies. ${ }^{11}$ This quantity shows the anomalous, "nonKorringa," behavior $1 / T_{1}=$ const in the cuprates. At these low frequencies we have to consider the collective, hydrodynamic response of the domain-wall fluid, which we expect to be determined by the dynamics of the domain-wall density, because this density is a conserved quantity in the model of Sec. II. Furthermore, thermodynamic information is needed where we can exploit the (extensive) literature. At this point the absence of large, long-range domain-wall interactions (Sec. II) becomes of central importance. Under this condition, the only interactions of thermodynamic significance have an entropic origin: if two walls collide, the meandering phase space is restricted, giving rise to long-range entropic repulsions. ${ }^{33}$ As Coppersmith et al., showed ${ }^{34}$ this implies that this system has a zero-temperature Kosterlitz-Thouless transition. At any finite temperature, free dislocations proliferate and correlation functions decay exponentially. After coarse graining, the free energy of the domain-wall liquid is equivalent to that of the $X Y$ model, except that the interaction parameter $(J)$ goes like temperature itself; as a resul the Kosterlitz-Thouless criterium never gets fulfilled at any finite temperature, since temperature drops out from the ratio $J / k_{B} T$.

Given the assumptions in Sec. II, our domain-wall liquid has to be of the same, dislocation-melted variety. In this case one expects standard hydrodynamical behavior. The wall density $\rho$ will obey a diffusion type equation of the form $\partial \rho / \partial t=D \nabla^{2} \rho$. Associated with the domain-wall density is then the hydrodynamic susceptibility ${ }^{37}$

$$
\chi_{\rho}^{\prime \prime}(q, \omega)=D \chi_{\rho} \omega \frac{q^{2}}{\omega^{2}+D^{2} q^{4}}
$$

where $\chi_{\rho}$ is the static density susceptibility. The diffusion constant is set by typical microscopic length and time scales. In the present case, we expect

$$
D \sim d^{2} \Gamma \sim T,
$$


the diffusion constant increases with temperature, because the average motion of the collision points (causing the diffusion) does so. The other quantity with potential temperature dependences is $\chi_{\rho}$ which contains the thermodynamical effects. As a trivial consequence of the work of Coppersmith et al. ${ }^{34}$ this quantity cannot have any sizeable temperature dependence. As we remarked above, in $X Y$ language, the susceptibility is a function of $\beta J$ and because $J$ itself is proportional to temperature for a domain-wall network dominated by entropic wall-wall interactions, the susceptibility becomes essentially temperature independent.

We can now address the NMR relaxation rate. In principle, the relationship between the domain-wall density susceptibility and the spin susceptibility is not trivial (the staggered order parameter is not a conserved quantity), but again this difference should not matter locally. This, together with the form factor, is lumped together into a (inconsequential) momentum cutoff $q_{c}$,

$$
\begin{aligned}
\frac{1}{T_{1}} & \sim \frac{T}{\omega_{0}} \int_{0}^{q_{c}} d^{2} q \chi_{\rho}^{\prime \prime}\left(q, \omega_{0}\right) \\
& \sim 2 \pi D \chi T \int_{0}^{q_{c}} d q \frac{q^{3}}{\omega_{0}^{2}+D^{2} q^{4}} \\
& \sim \frac{\pi \chi T}{2 D} \ln \left(1+\frac{D^{2} q_{c}^{4}}{\omega_{0}^{2}}\right) .
\end{aligned}
$$

Apart from logarithmic corrections coming from a possible temperature dependence of the momentum cutoff we find $1 / T_{1}$ to be temperature independent: the temperature dependence originating in the Bose factor is exactly canceled by the linear increase of the diffusion constant with temperature. We notice that this behavior of $1 / T_{1}$ is equivalent to the local dynamical spin susceptibility behaving as $\omega / T$ (Fig. 5).

Let us summarize the status of these results. Our analysis shows that if we assume that the charge and spin degrees of freedom in the copper-oxide planes of the high- $T_{c}$ superconductors form inhomogeneous phases that can be characterized in terms of reasonably homogeous domains separated by domain walls (a picture most relevant at low to moderate dopings), we find that for very reasonable parameters and $\mu=\mathscr{Q}(1)$ these wall fluctuation modes are important lowlying excitations whose temperature dependence recovers some of the important characteristic behavior of the high$T_{c}$ materials. Even if these fluctuations are not the origin of the characteristic behavior of the high- $T_{c}$ materials, they will still be important whenever $\mu$ is of order unity. If, on the other hand, there are inhomogeous phases with walls that have $\mu \ll 1$, then the walls are very stiff (so that, e.g., $c_{W}$ is large), and fluctuations become less important. In the next section, we will argue that whenever $\mu \gtrsim 1$, quantum fluctuation effects also become important at low temperature.

\section{DOMAIN WALLS AND QUANTUM MECHANICS}

So far, our discussion of the domain-wall dynamics has been based on classical fluctuation theory. Since the domain walls have a small mass it may be expected though that quantum mechanics plays an inportant role.

Let us first discuss some elementary facts about quantum wandering. The string Hamiltonian (7) can be quantized by requiring that $p_{q}=-i \hbar\left(\partial / \partial z_{q}\right)$,

$$
\begin{gathered}
H \Psi=E \Psi, \\
H=\int d q \hbar \omega_{q}\left(b_{q}^{\dagger} b_{q}+\frac{1}{2}\right),
\end{gathered}
$$

where

$$
\begin{gathered}
b_{q}^{\dagger}=\sqrt{\frac{\rho \omega_{q}}{2 \hbar}}\left(z_{q}-i \frac{p_{q}}{\rho \omega_{q}}\right), \\
b_{q}=\sqrt{\frac{\rho \omega_{q}}{2 \hbar}}\left(z_{-q}+i \frac{p_{-q}}{\rho \omega_{q}}\right), \\
{\left[b_{q}, b_{q^{\prime}}^{\dagger}\right]=\delta\left(q-q^{\prime}\right),}
\end{gathered}
$$

describe the quantized transverse sound "phonon" carrying momentum $q$ on the wall. The analogue of Eq. (2) for the equal time mean-square fluctuations in the perpendicular direction is now given by

$$
\begin{aligned}
\left\langle[z(\ell)-z(0)]^{2}\right\rangle & =\int_{-\pi / a}^{\pi / a} \frac{d q}{\pi}(1-\cos q \ell)\left\langle\left|z_{q}\right|^{2}\right\rangle \\
& =\frac{\hbar}{\pi \rho c_{W}} \int_{0}^{\pi / a} d q \frac{(1-\cos q \ell)}{q}\left(1+2 n_{q}\right) .
\end{aligned}
$$

At large temperatures the Bose factor dominates and one recovers the classical result Eq. (2) for equal times. At $T=0$, however, one has one factor of $1 / q$ less in the integral and the quantum wandering is therefore logarithmic

$$
\left\langle[z(\ell)-z(0)]^{2}\right\rangle_{Q}=\frac{\hbar}{\pi \rho c_{W}} \ln \left(\frac{\ell \pi e^{\gamma}}{a}\right),
$$

where $\gamma \simeq 0.577$ is Euler's constant. At a given length scale $\ell$, the temperature at which a crossover from classical to quantum behavior occurs is of the order of $k_{B} T \sim \pi \hbar c_{W} / \ell$. Alternatively, at a fixed temperature the wandering is classical at length scales larger than $\hbar c_{W} / k_{B} T$, and quantum mechanical at length scales much smaller than this value.

Equating the zero-temperature mean-square fluctuation to $d^{2}$ yields, together with (6), the quantum collision length

$$
\ell_{c}^{Q}=\frac{a}{\pi e^{\gamma}} e^{\pi^{2} / \mu},
$$

and the associated energy scale

$$
\begin{aligned}
\hbar \Gamma_{Q} & =\frac{\hbar \pi c_{W}}{\ell_{c}^{Q}} \\
& =\frac{\pi^{2} \hbar c_{W} e^{\gamma}}{a} e^{-\pi^{2} / \mu} .
\end{aligned}
$$

We can now make the criterium for the relevance of quantum mechanics precise: this is controlled by $\mu$, the appropriate dimensionless parameter for the present problem defined in 
Eq. (6). If $\mu \ll 1$, the quantum fluctuations are exponentially suppressed and the problem remains classical down to the lowest temperatures. If $\mu \gg 1$ quantum fluctuations are dominating to such an extent that they are important even at the momentum cutoff so that the present long-wavelength analysis looses its validity. When $\mu$ is of order unity, however, a crossover occurs from a high temperature classical regime to a low-temperature quantum-dominated regime at a reasonable temperature of order of the intrinsic energy scale of the problem.

In fact, in this respect the situation is comparable to that in the helium. The so-called de Boer parameter ${ }^{27}$ which measures the strength of quantum effects for atomic motion in crystals is defined as the ratio $(\hbar / a)(m U)^{-1 / 2}$ where $m$ is the mass of the atoms, $a$ the lattice parameter, and $U$ a typical interaction energy. Of the nobel gases, only for $\mathrm{He}$ is this parameter of order unity, explaining why it can be regarded as a classical system at high temperatures while it forms quantum fluids and crystals at low temperatures. ${ }^{27}$ If one inserts $\rho=m / a$ and $\Sigma=U / a$ one recovers, together with (6), our expression (6) for $\mu$, showing that we may think of $\mu$ as the analog of the de Boer parameter.

According to the neutron data for the doped $\mathrm{La}_{2} \mathrm{CuO}_{4}$ system, $\mu$ seems to increase from $\sim 1 / 2$ at low dopings $(x=0.04)^{26}$ to $\sim 2$ at superconducting concentrations $\left(x=0.14,{ }^{18,36}\right)$ roughly consistent with the expected increase of the domain-wall density due to the increasing hole density $\left(\mu \sim 1 / d^{2} \sim x^{2}\right)$. The suggestion we read from this is the following: in the former case, the quantum fluctuations are not strong enough to prevent classical ordering as in the nickelate. On the other hand, at higher concentrations we expect a crossover to occur from a high-temperature classical regime to a low-temperature quantum regime because $\mu \simeq \pi$, in direct analogy with He. In particular, we envision the existence of a quantum domain-wall liquid, i.e., a phase with strong domain-wall correlations on short-time scales but liquidlike properties on long-time scales. The crossover to quantum behavior implies that the previously found form of the susceptibilities is valid only above the scale $\Gamma_{Q}$. The possible behavior below this crossover scale will be discussed further below.

\section{DISCUSSION}

In making up a balance of what we have reached, we observe that the central assumption underlying our results is in our treatment of domain walls as robust, pre-existing objects. It is probably more realistic to view this system of walls of Sec. II as a strong-coupling regime of which $\mathrm{La}_{2-x} \mathrm{Sr}_{x} \mathrm{NiO}_{4}$ (and possibly the underdoped cuprates) is the limiting behavior. Its weak-coupling counterpart would be more like a system of essentially free holes, while the high$T_{c}$ superconductors should probably be thought of in terms of intermediate-couplings. The recent results ${ }^{1}$ for the $t-J$ model suggest that this latter intermediate-coupling regime could be quite large.

As usual, the strategy is to understand both the weak- and strong-couplings regimes and to find out how to connect the two. In rough outline we have sketched the nature of this strong-coupling regime in the above. This amounted to keeping the domain walls as robust objects, while they were sub- jected to "transverse" meandering fluctuations. For decreasing coupling strength the walls should start to loose their integrity and one imagines that the remnants of these domain walls live as (many particle) resonances in a bath of fermionic excitations. One would like to think of these resonances as strands of domain-wall of some characteristic length and lifetime. If this lifetime is sufficiently long (compared to $1 / \Gamma$ ) and this length sufficiently large (compared to $l_{c}$ ) it would still make sense to approach this problem from the strong-coupling side, in the same sense that Heisenberg models are relevant for itinerant magnets.

What is the characteristic binding energy of the domain walls? In this regard, doped $\mathrm{La}_{2} \mathrm{NiO}_{4}$ is illuminating. Doping induces gap states in the optical conductivity. The threshold of these absorptions is characterized by a "polaronic resonance" at $0.5 \mathrm{eV}^{41,42}$ Since we know that the holes are bound to domain walls in this case, it is tempting to interpret these resonances to correspond with the process of exciting holes out of the domain walls. Assuming harmonic potentials, we infer a binding energy $\sim 0.25 \mathrm{eV}$. Assuming that charged domain walls are also relevant for the underdoped cuprates, we infer from the similar polaronic peak in the optical conductivity of the cuprates ${ }^{43}$ that the binding energy of the holes to the domain walls is now of order of $0.1 \mathrm{eV}$. Apparently, this "polaronic resonance" survives if doping increases, although in the metals it is accompanied by a Drude-like feature with a weight growing rapidly with doping. ${ }^{44}$ This might be interpreted in terms of domain-wall "resonances" coexisting with a particlelike bath.

If this interpretation is correct, it implies that domain walls get destroyed by thermal fluctuations at temperatures of at most $1000 \mathrm{~K}$ - together with the de Boer criterium this leaves a relatively small window in temperature for the observation of the dynamics we discussed in the previous sections.

It is also interesting to point out that the relatively small string tension $\Sigma$ as estimated for parameters relevant for the cuprates, indicates that walls are not only rather mobile, but also rather wide, just like the small soliton mass in the SuSchrieffer-Heeger model of polyacetylene ${ }^{45}$ results from the large width of the soliton. As discussed in the introduction, this implies that the relative weight of higher harmonics of the domain-wall correlations will be small.

Let us now consider the dc transport. Suppose we would consider for a moment the domain walls as pre-existing, unbreakable entities. Such a charged domain-wall liquid could still be an electrical conductor, but we would then expect a domain-wall motion-type transport, analogous to that of a polymer liquid, ${ }^{46}$ with a resistivity that decreases with increasing temperature. The opposite is true in reality, pointing at a serious shortcoming of a scenario in which all holes are tightly bound to domain walls: it forces us to consider the possilibility of exchange with particles and to relax the assumption that walls are unalterable pre-existing entities. For instance, a simple alternative scenario for the behavior of the dc resistivity could follow the lines of the marginal-Fermiliquid ideas of Varma et al. ${ }^{13,16}$ or the paramagnon approach of Pines and co-workers: ${ }^{14}$ it is assumed that the electric current is carried by fermionic excitations which are scattered by the anomalous fluctuations which we would interpret as domain-wall fluctuations. 
We now turn to low temperatures. In Sec. IV we argued that the high- $T_{c}$ superconductors are like He, in the sense that at some reasonable temperature a crossover follows from a classical- to a low-temperature quantal regime. The shortcomings of the assumptions in Sec. II are most easily demonstrated in the latter.

Regardless the interpretation of the so-called spin gaps, it appears that at least at higher dopings ${ }^{47}$ the spin system in the cuprates is at zero temperature in an incompressible liquid state if not only because of the superconductivity. Even if the domain walls would form an (incompressible) quantum liquid, one would still expect to find low-energy excitations associated with the spin waves in the domains, if we take assumption (iii) of Sec. II literally (all additional, hole induced spin fluctuation coming from the wall motions). We argued that the softness of the former could mask the latter if both fluids are compressible. However, at zero temperature it would imply that the domain-wall liquid would coexist with a Néel solid, and this possibility is clearly ruled out by experiment. In this regard, the observations in a recent paper by Tsunetsugu et al $^{48}$ are interesting. It is argued that the spins in the domains would remain in an incompressible state for small but finite interdomain spin-spin interaction, at least if the domain walls are in an ordered state.

A static ordering of the domain walls seems also excluded experimentally and this implies a serious problem with regards to assumption (i) of Sec. II, that the domain walls behave as Gaussian strings. The quantum fluctuation of the gaussian strings is only weakly (logarithmically) divergent and the question arises whether this can prevent the existence of a striped phase order parameter at zero temperature in the dense system. ${ }^{33}$ Some insight into this question can be gained by the following argument, analogous to the Lindemann criterium for the melting of solids: we start out at $\mu=0$ with an ordered state and we ask if the mean-square fluctuation of a single wall can become of order the domainwall separation $d$ before $\mu$ reaches unity. The presence of a striped phase order parameter changes the properties of a single string. The string is now constrained to move in a static tube of finite width, which removes low-lying modes from the spectrum - e.g., if we model this tube constraint with a harmonic potential, the string sound modes would simply acquire a mass. More generally, the transversal extent of the wave function belonging to string mode $q$ is typically $z_{q}=\sqrt{\hbar / \rho a c_{W} q}$. If mode couplings due to collisions with the tube are neglected, the influence of the tube is to remove modes with $z_{q}>d$ from the sound spectrum. This implies a low momentum cutoff in the integral Eq. (31) and recalcu- lating the domain-wall fluctuation we find $\left\langle(\Delta z)^{2}\right\rangle / d^{2}$ $\simeq\left(\mu / \pi^{2}\right) \ln \mu$. This simple argument shows that quantum melting of the striped phase can only happen if the Gaussian theory of the isolated walls looses its validity $(\mu>\pi)$, because otherwise the width of the walls is small compared to their separation. ${ }^{49}$

This might again be taken as an indication for the importance of fluctuations involving the exchange with a particle bath. The action of the Gaussian string is proportional to the total surface area of the string in space time. On the other hand, if the string can freely exchange with a particle bath, with the particles being the constituents of the string, the surface area invariant vanishes and the physics is governed by higher-order invariants. The next possibility leads to the so-called "extrinsic curvature" surfaces/quantum strings ${ }^{50}$ which are characterized by a particlelike spectrum $\omega_{q} \sim q^{2}$, and accordingly by a much stronger (algebraic) divergency, curing the ordering problem discussed in the previous paragraph. ${ }^{51}$

Obviously the most severe problem is that the lowfrequency, low-temperature regime in the high- $T_{c}$ superconductors is dominated by fermionic excitations which behave in many ways as normal electrons. The challenge remains to find a way to reconcile these fermions with the string liquid possibly realized at shorter times and higher temperatures. The recent work ${ }^{1}$ showing the coexistence of strong domainwall correlations with some of the more quasiparticlelike properties of correlated fermions in the $t-J$ model shows that the same dichotomy arises in theoretical models. Understanding this complementarity will be an important object for future study.

Note added after submission: After submission of this paper we learned that the charged domain-wall striped phase has actually been observed in a statically ordered form in a high- $T_{c}$ cuprate. Because of well understood commensuration effects the charged domain walls freeze out at the special doping concentration of $x=1 / 8$, in the low-temperature tetragonal phase [J. M. Tranquada, B. J. Sternlieb, J. D. Axe, Y. Nakamura, and S. Uchida, Nature (London) 375, 561 (1995)].

\section{ACKNOWLEDGMENTS}

The work by M.L.H. at Leiden University was supported by the Netherlands Foundation for Fundamental Research on Matter (FOM) and his work at Rutgers University by the Netherlands Organization for Scientific Research (NWO). J.Z. aknowledges financial support by the Dutch Royal Academy of sciences (KNAW).
*Present address: Serin Physics Laboratory, Rutgers University, P.O. Box 849, Piscataway, New Jersey 08855.

${ }^{1}$ P. Prelovsek and X. Zotos, Phys. Rev. B 47, 5984 (1993); P. Prelovsek and I. Sega, ibid. 49, 15241 (1994).

${ }^{2}$ V. J. Emery, S. Kivelson, and H-Q. Lin, Phys. Rev. Lett. 64, 475 (1990); V. J. Emery and S. Kivelson, Physica C 209, 597 (1993).

${ }^{3}$ J. Zaanen and O. Gunnarson, Phys. Rev. B 40, 7391 (1989); H. J. Schulz, Phys. Rev. Lett. 64, 1445 (1990); M. Kato, K. Machida, H. Nakanishi, and M. Fujita, J. Phys. Soc. Jpn. 59, 1047 (1990); M. Inui and P. B. Littlewood, Phys. Rev. B 44, 4415 (1991); J.
A. Vergés, F. Guinea, and E. Louis, ibid. 46, 3562 (1992).

${ }^{4}$ D. Poilblanc and T. M. Rice, Phys. Rev. B 39, 9749 (1989).

${ }^{5}$ T. Giamarchi and C. Lhuillier, Phys. Rev. B 43, 12943 (1991).

${ }^{6}$ G. An and J. M. J. van Leeuwen, Phys. Rev. 44, 9410 (1991); H. J. M. van Bemmel, D. F. B. ten Haaf, W. van Saarloos, J. M. J. van Leeuwen, and G. An, Phys. Rev. Lett. 72, 2442 (1994).

${ }^{7}$ U. Löw, V. J. Emery, K. Fabricius, and S. A. Kivelson, Phys. Rev. Lett. 72, 1918 (1994).

${ }^{8}$ J. M. Tranquada, D. J. Buttrey, V. Sachan, and J. E. Lorenzo, Phys. Rev. Lett. 73, 1003 (1994). 
${ }^{9}$ J. Zaanen and P. B. Littlewood, Phys. Rev. B 50, 7222 (1994).

${ }^{10}$ J. H. Cho, F. C. Chou, and D. C. Johnston, Phys. Rev. Lett. 70, 222 (1993).

${ }^{11}$ V. Barzykin, D. Pines, and D. Thelen, Phys. Rev. B 50, 16052 (1994)

${ }^{12}$ R. E. Walstedt, B. S. Shastry, and S.-W. Cheong, Phys. Rev. Lett. 72, 3610 (1994).

${ }^{13}$ P. B. Littlewood, in Strongly Interacting Fermions and High $T_{c}$ Superconductivity, edited by B. Douçot and J. Zinn-Justin (North-Holland, Amsterdam, 1995), p. 69.

${ }^{14}$ P. Monthoux, A. V. Balatsky, and D. Pines, Phys. Rev. Lett. 67, 3448 (1991); Phys. Rev. B 46, 14803 (1992); P. Monthoux and D. Pines, ibid. 49, 4261 (1994).

${ }^{15}$ P. W. Anderson, in Proceedings of the International Workshop on Electronic Properties and Mechanisms in High- $T_{c}$ Superconductors, edited by K. Kadowaki and T. Oguchi (Elsevier, Amsterdam, 1991).

${ }^{16}$ C. M. Varma, P. B. Littlewood, S. Schmitt-Rink, E. Abrahams, and A. E. Ruckenstein, Phys. Rev. Lett. 63, 1996 (1989); P. B. Littlewood and C. M. Varma, J. Appl. Phys. 69, 4979 (1991).

${ }^{17}$ S.-W. Cheong, G. Aeppli, T. E. Mason, H. Mook, S. M. Hayden, P. C. Canfield, Z. Fisk, K. N. Clausen, and J. L. Martinez, Phys. Rev. Lett. 67, 1791 (1991); G. Shirane, R. J. Birgeneau, Y. Endoh, and M. A. Kastner, Physica B 197, 158 (1994).

${ }^{18}$ T. E. Mason, G. Aeppli, and H. A. Mook, Phys. Rev. Lett. 68, 1414 (1992).

${ }^{19}$ B. Shraiman and E. D. Siggia, Phys. Rev. Lett. 62, 1546 (1989); D. M. Frenkel, R. J. Gooding, B. I. Shraiman, and E. D. Siggia, Phys. Rev. B 41, 350 (1990); T. Dombre, J. Phys. (Paris) 51, 847 (1990).

${ }^{20}$ P. B. Littlewood, J. Zaanen, H. Monien, and G. Aeppli, Phys. Rev. B 48, 487 (1993).

${ }^{21}$ R. J. Jelitto, Phys. Status Solidi B 147, 391 (1988); N. Bulut, D. Hone, D. J. Scalapino, and N. E. Bickers, Phys. Rev. B 41, 1797 (1990).

${ }^{22}$ J. P. Lu, Q. Si, J. H. Kim, and K. Levin, Phys. Rev. Lett. 65, 2466 (1990); Y. Zha, Q. Si, and K. Levin, Phys. Rev. B 47, 9055 (1993)

${ }^{23}$ V. I. Anisimov, M. A. Korotin, J. Zaanen, and O. K. Andersen, Phys. Rev. Lett. 68, 345 (1992).

${ }^{24}$ C. H. Chen, S.-W. Cheong, and A. S. Cooper, Phys. Rev. Lett. 71, 2461 (1993); Th. Strangfeld, K. Westerholt, and H. Bach, Physica C 183, 1 (1991); X.-X. Bi and P. C. Ecklund, Phys. Rev. Lett. 70, 2625 (1993).

${ }^{25}$ S. M. Hayden, G. H. Lander, J. Zaretsky, P. J. Brown, C. Stassis, P. Metcalf, and J. M. Honig, Phys. Rev. Lett. 68, 1061 (1992).

${ }^{26}$ S. M. Hayden, G. Aeppli, H. Mook, D. Rytz, M. F. Hundley, and Z. Fisk, Phys. Rev. Lett. 66, 821 (1991); B. Keimer, R. J. Birgeneau, A. Cassanho, Y. Endoh, R. W. Erwin, M. A. Kastner, and G. Shirane, ibid. 67, 1930 (1991).

${ }^{27}$ A. F. Andreev, in Progress in Low Temperature Physics, edited by D. F. Brewer (North-Holland, Amsterdam, 1982), Vol. VIII.

${ }^{28}$ Since we neglect lattice anisotropies, domain-wall fluctuations depend on the string tension only. If the lattice anisotropy is important, the string tension has to be replaced by a string stiffness. See, e.g., P. Nozières, in Solids far from Equilibrium, edited by C. Godrèche (Cambridge University Press, Cambridge, 1991).
${ }^{29}$ W. L. McMillan, Phys. Rev. B 12, 1187 (1975); 12, 1197 (1975); G. Grüner, Rev. Mod. Phys. 60, 1129 (1988).

${ }^{30}$ H. Neuberger and T. Ziman, Phys. Rev. B 39, 2608 (1989).

${ }^{31}$ V. L. Pokrovsky and A. L. Talapov, Phys. Rev. Lett. 42, 65 (1979).

${ }^{32}$ M. den Nijs, in Phase Transitions and Critical Phenomena, edited by C. Domb and J. L. Lebowitz (Academic, London, 1988), Vol. 12, p. 220.

${ }^{33}$ M. E. Fisher and D. S. Fisher, Phys. Rev. B 25, 3192 (1982).

${ }^{34}$ S. N. Coppersmith, D. S. Fisher, B. I. Halperin, P. A. Lee, and W. F. Brinkman, Phys. Rev. B 25, 349 (1982).

${ }^{35}$ The factor 2 in Eq. (5) is introduced largely for convenience; intuitively, the factor 2 is not unreasonable, since the lowest eigenmode of a classical string between nodes a distance $\ell_{c}$ apart corresponds to a wavelength $2 \ell_{c}$.

${ }^{36}$ The $\mu$, indirectly inferred from optical conductivity in $\mathrm{YBa}_{2} \mathrm{Cu}_{3} \mathrm{O}_{7}$, would be about $\pi$ : Z. Schlesinger, R. T. Collins, F. Holtzberg, C. Feild, S. H. Blanton, U. Welp, G. W. Crabtree, Y. Fang, and J. Z. Liu, Phys. Rev. Lett. 65, 801 (1990).

${ }^{37}$ D. Forster, Hydrodynamic Fluctuations, Broken Symmetry, and Correlation Functions (Addison-Wesley, Reading, MA, 1975).

${ }^{38}$ S. M. Hayden, T. E. Mason, G. Aeppli, H. A. Mook, S.-W. Cheong, and Z. Fisk, in Phase Separation in Cuprate Superconductors (World Scientific, Singapore, 1993).

${ }^{39}$ S. Sachdev and J. Ye, Phys. Rev. Lett. 69, 2411 (1992); A. V. Chubukov and S. Sachdev, ibid. 71, 169 (1993).

${ }^{40}$ A. J. Millis, H. Monien, and D. Pines, Phys. Rev. B 42, 167 (1990).

${ }^{41}$ S. Chakravarty and R. Orbach, Phys. Rev. Lett. 64, 224 (1990).

${ }^{42}$ T. Ido, K. Magoshi, H. Eisaki, and S. Uchida, Phys. Rev. B 44, 12094 (1991).

${ }^{43}$ G. A. Thomas, D. H. Rapkine, S. L. Cooper, S.-W. Cheong, A. S. Cooper, L. F. Schneemeyer, and J. V. Waszczak, Phys. Rev. B 45, 2474 (1992).

${ }^{44}$ S. Uchida, T. Ido, H. Takagi, T. Arami, Y. Tokura, and S. Tajima, Phys. Rev. B 43, 7942 (1991); S. L. Cooper, G. A. Thomas, J. Orenstein, D. H. Rapkine, M. Capizzi, T. Timusk, A. J. Millis, L. F. Schneemeyer, and J. V. Waszczak, ibid. 44, 11358 (1989); J. Orenstein, G. A. Thomas, A. J. Millis, S. L. Cooper, D. H. Rapkine, T. Timusk, L. F. Schneemeyer, and J. V. Waszczak, Phys. Rev. B 42, 6342 (1990).

${ }^{45}$ A. J. Heeger, S. Kivelson, J. R. Schrieffer, and W.-P. Su, Rev. Mod. Phys. 60, 781 (1988).

${ }^{46}$ P. G. de Gennes, Scaling Concepts in Polymer Physics (Cornell University Press, Ithaca, 1979).

${ }^{47}$ T. E. Mason, G. Aeppli, S. M. Hayden, A. P. Ramirez, and H. A. Mook, Phys. Rev. Lett. 71, 919 (1994).

${ }^{48}$ H. Tsunetsugu, M. Troyer, and T. M. Rice, Phys. Rev. B 51, 16456 (1995).

${ }^{49}$ These arguments might in fact apply to doped $\mathrm{La}_{2} \mathrm{NiO}_{4}$. A recent spectroscopic study suggests severe quantum meandering fluctuations on short length scales [E. Erpel (unpublished)].

${ }^{50}$ See, e.g., R. Lipowsky, Nature (London) 349, 475 (1991) or G. Forgacs, R. Lipowsky, and Th. M. Nieuwenhuizen, in Phase Transitions and Critical Phenomena, edited by C. Domb and J. L. Lebowitz (Academic, London, 1991), Vol. 14, p. 135.

${ }^{51}$ This might be the continuum limit of the mechanism for quantum melting as proposed in H. Viertiö and T. M. Rice, J. Phys. Condens. Matter 6, 7090 (1994). 